University of Warwick institutional repository: http://go.warwick.ac.uk/wrap This paper is made available online in accordance with publisher policies. Please scroll down to view the document itself. Please refer to the repository record for this item and our policy information available from the repository home page for further information.

To see the final version of this paper please visit the publisher's website. Access to the published version may require a subscription.

Author(s): T. D. Veal, P. H. Jefferson, L. F. J. Piper, C. F. McConville, T. B. Joyce, P. R. Chalker, L. Considine, Hai Lu, and W. J. Schaff Article Title: Transition from electron accumulation to depletion at InGaN surfaces

Year of publication: 2006

Link to published version: http://dx.doi.org/ 10.1063/1.2387976

Publisher statement: None 


\title{
Transition from electron accumulation to depletion at InGaN surfaces
}

\author{
T. D. Veal, ${ }^{\text {a) }}$ P. H. Jefferson, L. F. J. Piper, and C. F. McConville ${ }^{\text {b) }}$ \\ Department of Physics, University of Warwick, Coventry, CV4 7AL, United Kingdom \\ T. B. Joyce and P. R. Chalker \\ Department of Materials Science and Engineering, University of Liverpool, Liverpool, L69 3BX, United \\ Kingdom \\ L. Considine \\ Thomas Swan and Co. Ltd., Harston, Cambridge CB2 5NX, United Kingdom \\ Hai Lu and W. J. Schaff \\ Department of Electrical and Computer Engineering, Cornell University, Ithaca, New York 14853
}

(Received 16 August 2006; accepted 28 September 2006; published online 14 November 2006)

\begin{abstract}
The composition dependence of the Fermi-level pinning at the oxidized (0001) surfaces of $n$-type $\mathrm{In}_{x} \mathrm{Ga}_{1-x} \mathrm{~N}$ films $(0 \leqslant x \leqslant 1)$ is investigated using $\mathrm{x}$-ray photoemission spectroscopy. The surface Fermi-level position varies from high above the conduction band minimum (CBM) at InN surfaces to significantly below the $\mathrm{CBM}$ at $\mathrm{GaN}$ surfaces, with the transition from electron accumulation to depletion occurring at approximately $x=0.3$. The results are consistent with the composition dependence of the band edges with respect to the charge neutrality level. () 2006 American Institute of Physics. [DOI: 10.1063/1.2387976]
\end{abstract}

The surface of InN thin films has recently been found to exhibit electron accumulation, with the surface Fermi level lying far above the conduction band edge. ${ }^{1,2}$ Similarly, at InAs surfaces and interfaces, the Fermi level has long been known to lie above the conduction band edge, ${ }^{3}$ resulting in $n$-InAs/metal contacts exhibiting Ohmic behavior with electron accumulation layers rather than Schottky barriers with depletion space charge regions. Moreover, it has been shown that this is also true for In-rich $\operatorname{In}_{x} \mathrm{Ga}_{1-x}$ As alloys, with the barrier height varying as $\Phi_{B}=0.95-1.90 x+0.90 x^{2}$, implying that the transition from positive to negative "barrier height" occurs at $x \sim 0.8 .{ }^{4,5}$ However, no investigation has been reported of the transition in InGaN alloys from electron accumulation at InN surfaces to electron depletion at GaN surfaces.

In this letter, the composition dependence of the Fermilevel position with respect to the band edges at $\operatorname{In}_{x} \mathrm{Ga}_{1-x} \mathrm{~N}$ surfaces is investigated using high-resolution $\mathrm{x}$-ray photoemission spectroscopy (XPS). XPS has previously been used to study the surface electronic properties of a wide range of semiconductors. ${ }^{6}$ While clean surfaces of both $\mathrm{GaN}$ and $\mathrm{InN}$ have previously been obtained outside the growth chamber, ${ }^{7,8}$ surface preparation remains very difficult and would require a different method to be optimized for each $\mathrm{In}_{x} \mathrm{Ga}_{1-x} \mathrm{~N}$ composition. Consequently, the composition dependence of the surface Fermi-level pinning has been studied in the presence of the native oxide on the $\operatorname{In}_{x} \mathrm{Ga}_{1-x} \mathrm{~N}(0001)$ surfaces. Knowledge of the surface Fermi level is important for surface sensitive devices, such as chemical and biological sensors, where an InGaN active layer is exposed to the environment. Indeed, the potential of InN's surface for use as a sensor has already been demonstrated by a study of its electrical response to chemical exposures. ${ }^{9}$

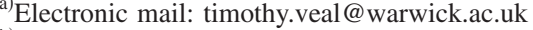

${ }^{b)}$ Electronic mail: c.f.mcconville@warwick.ac.uk
}

The GaN and $\mathrm{In}_{0.14} \mathrm{Ga}_{0.86} \mathrm{~N}$ samples were grown on $c$-axis sapphire by metal-organic chemical vapor deposition (MOCVD). An initial low temperature GaN buffer layer was deposited at $550{ }^{\circ} \mathrm{C}$ with a subsequent increase in substrate temperature to $1050{ }^{\circ} \mathrm{C}$ for the $\mathrm{GaN}$ epilayer deposition. The $\mathrm{In}_{0.14} \mathrm{Ga}_{0.86} \mathrm{~N}$ layer was grown at a reduced substrate temperature of $830{ }^{\circ} \mathrm{C}$. The $\mathrm{In}_{x} \mathrm{Ga}_{1-x} \mathrm{~N}$ samples for which 0.43 $\leqslant x \leqslant 1.00$ were grown on $c$-axis sapphire by molecularbeam epitaxy (MBE). An initial nitridation of the sapphire at $200{ }^{\circ} \mathrm{C}$ was followed by the deposition at $800^{\circ} \mathrm{C}$ of a 10-nm-thick AlN buffer layer and a GaN buffer layer grown at $750{ }^{\circ} \mathrm{C} .{ }^{10}$ The $\mathrm{In}_{x} \mathrm{Ga}_{1-x} \mathrm{~N}$ layers were grown to thicknesses between 100 and $500 \mathrm{~nm}$ at temperatures of between 480 and $600{ }^{\circ} \mathrm{C}$. The free electron densities in the MBEgrown samples range from $3 \times 10^{19}$ to $1 \times 10^{18} \mathrm{~cm}^{-3}$, with electron mobilities ranging from 20 to $950 \mathrm{~cm}^{2} \mathrm{~V}^{-1} \mathrm{~s}^{-1}$. The composition of the $\operatorname{In}_{x} \mathrm{Ga}_{1-x} \mathrm{~N}$ layers was determined by $\mathrm{x}$-ray diffraction.

XPS analysis was performed using a Scienta ESCA300 spectrometer at the National Centre for Electron Spectroscopy and Surface Analysis, Daresbury Laboratory, UK. This consists of a rotating anode $\mathrm{Al} \mathrm{K} \alpha$ x-ray source ( $h \nu$ $=1486.6 \mathrm{eV}$ ), x-ray monochromator, and $300 \mathrm{~mm}$ mean radius spherical-sector electron energy analyzer with parallel electron detection system. The analyzer was operated with $0.8 \mathrm{~mm}$ slits and at a pass energy of $150 \mathrm{eV}$. Gaussian convolution of the analyzer broadening with an effective linewidth of $0.27 \mathrm{eV}$ for the $\mathrm{x}$-ray source gives an effective instrument resolution of $0.45 \mathrm{eV}$. The Fermi-level position (zero of the binding energy scale) was calibrated using the Fermi-edge of an ion bombarded silver reference sample which is regularly used to calibrate the spectrometer. The valence band maximum (VBM) is determined by linearly extrapolating the leading edge of the valence band to the base line in order to account for the instrumental resolutioninduced tail. ${ }^{11}$

The In $3 d$, Ga $2 p$, Ga $3 d, \mathrm{~N} 1 s, \mathrm{O} 1 s$, and $\mathrm{C} 1 s$ core levels were investigated with XPS to determine the nature of 


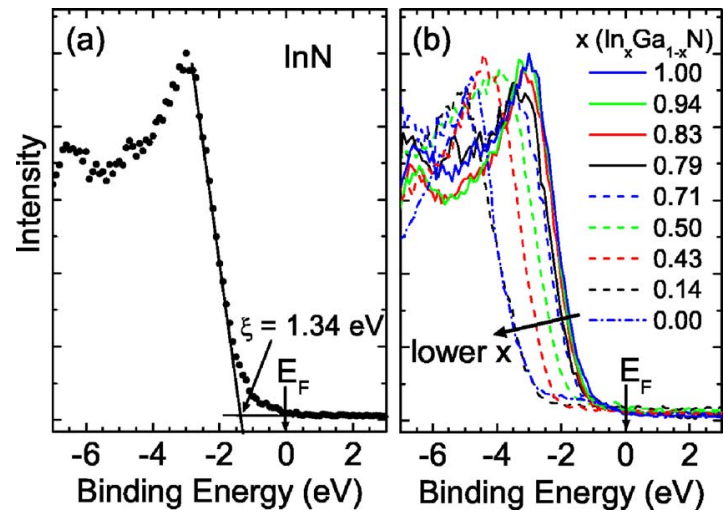

FIG. 1. (Color online) (a) Valence band XPS spectrum of InN in the region of the valence band maximum. The binding energy scale is with respect to the Fermi level $E_{F}$. The valence band maximum occurs at the intersection of a line fit to the linear portion of the leading edge and the extended background line between the valence band maximum and the Fermi level. The valence band maximum is estimated to lie $1.34 \pm 0.20 \mathrm{eV}$ below the surface Fermi level. (b) The valence band XPS spectra of $\operatorname{In}_{x} \mathrm{Ga}_{1-x} \mathrm{~N}$ alloys for 0 $\leqslant x \leqslant 1$. The binding energy scale is with respect to the Fermi level $E_{F}$.

the native oxide and other contamination on the $\operatorname{In}_{x} \mathrm{Ga}_{1-x} \mathrm{~N}$ surfaces. The native oxide consisted of group-III-O species, with no evidence of $\mathrm{N}-\mathrm{O}$ bonding. Additionally, adventitious oxygen and hydrocarbons were also present on top of the native oxide. The contamination layer thicknesses were estimated from the XPS results to be between 1.5 and $2.5 \mathrm{~nm}$ on the various InGaN samples.

A photoemission spectrum from $\mathrm{InN}$ in the region of the valence band maximum is shown in Fig. 1(a). The linear extrapolation of the leading edge of the valence band emission and the base line are also shown. The intersection of these two lines indicates that the VBM is at $1.34 \mathrm{eV}$ below the surface Fermi level. Since InN has a band gap of $0.65 \mathrm{eV}$ at room temperature, this indicates that the surface Fermi level is $0.69 \mathrm{eV}$ above the conduction band minimum $(\mathrm{CBM})$, consistent with previous studies employing highresolution electron-energy-loss spectroscopy and ultraviolet photoemission spectroscopy. ${ }^{2,12,13}$ Valence band photoemission spectra from a range of $\mathrm{InGaN}$ alloys are shown in Fig. 1(b). As the Ga content is increased in the alloy, the valence band maximum shifts further away from the surface Fermi level. When the change in band gap with composition is considered, this corresponds initially to a lowering of the surface Fermi level in the conduction band and then to the surface Fermi level moving below the CBM.

The separation between the CBM and the surface Fermi level or the barrier height (analogous to the Schottky barrier height of a metal/semiconductor contact) has been determined from the photoemission data across the range of alloy compositions and is shown in Fig. 2. The barrier height is positive (negative) for Ga-rich (In-rich) alloys, corresponding to the surface Fermi level lying below (above) the CBM. The composition dependence of the barrier height is estimated by the least squares method to be $\Phi_{B}=0.53-2.1 x$ $+0.95 x^{2}$ for $\operatorname{In}_{x} \mathrm{Ga}_{1-x} \mathrm{~N}$, suggesting that the surface Fermi level coincides with the CBM and the barrier height is zero at $x=0.29$. This composition does not necessarily coincide with the transition from surface electron depletion to accumulation as the nature of the space charge region is determined in a particular sample by whether the bulk Fermi level is above or below the surface Fermi level. However, for the

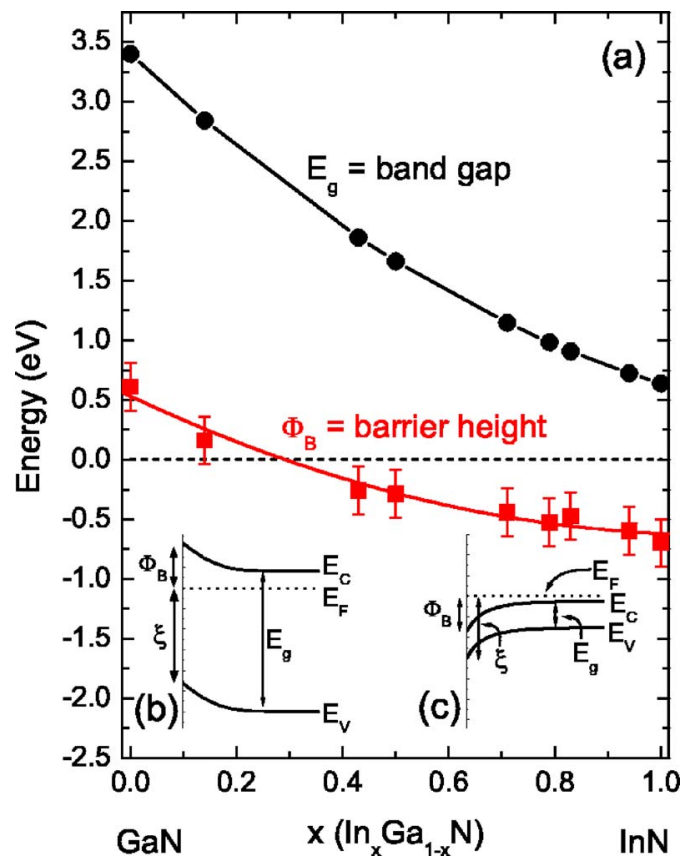

FIG. 2. (Color online) (a) Variation of band gap $E_{g}$ and "barrier height" $\Phi_{B}$ (the separation between the Fermi level and the CBM) at the $\operatorname{InGaN}(0001)$ surfaces with varying In concentration $x$ in the presence of the native oxide. The band gap points are for a bowing parameter of $1.4 \mathrm{eV}$ (Ref. 14). The barrier heights $\left(\Phi_{B}\right)$ are derived by subtracting the experimental surface Fermi level to VBM separation $(\xi)$ from the band gap $\left(\Phi_{B}=E_{g}-\xi\right)$. Positive values of $\Phi_{B}$ mean Fermi-level pinning in the band gap, while negative values of $\Phi_{B}$ mean Fermi-level pinning within the conduction band (see band schemes below). The line is a second order polynomial least squares fit to the $\Phi_{B}$ data points (solid line). The dashed line indicates the zero of energy. Insets (b) and (c) depict the upward band bending in a depletion layer at a $\mathrm{GaN}$ surface and the downward band bending in an accumulation layer at an $\mathrm{InN}$ surface, respectively.

MOCVD-grown GaN and $\mathrm{In}_{0.14} \mathrm{Ga}_{0.86} \mathrm{~N}$ samples studied here, calculations of the bulk Fermi level corresponding to the Hall-effect-measured free electron densities indicate that it is above the surface Fermi level; a surface depletion layer is present, as previously observed at the surface of MBEgrown $\mathrm{GaN} .{ }^{15}$ And for the samples with $x \geqslant 0.43$, the bulk Fermi level is below the surface Fermi level; surface electron accumulation is exhibited.

The observed variation of surface Fermi-level position is consistent with the alloy composition dependence of the band edges with respect to the charge neutrality level or branch-point energy $\left(E_{B}\right)$, where the interface induced gap states (IFIGS) change their charging character from donor to acceptor type. ${ }^{24}$ The band edges and the observed surface Fermi-level variation are shown with respect to $E_{B}$ in Fig. 3. For the In-rich alloys, the surface Fermi level is below $E_{B}$, allowing donor-type IFIGS in the vicinity of $E_{B}$ to be empty and donate electrons into the accumulation layer. For the Ga-rich alloys, the surface Fermi level is above $E_{B}$ such that acceptor-type IFIGS close to $E_{B}$ are occupied, negatively charged, and contribute to the electron depletion layer. The observed surface Fermi level is also influenced by charge transfer between the semiconductor and the more electronegative native oxide and less electronegative group III adlayers. ${ }^{5,24}$ Finally, the surface Fermi-level position determined by the XPS is an average over a finite depth from the surface due to the exponential form of the attenuation length of the photoelectrons. For degenerately doped InN, the short screening lengths mean that the bands bend rapidly as a AIP license or copyright; see http://apl.aip.org/apl/copyright.jsp 


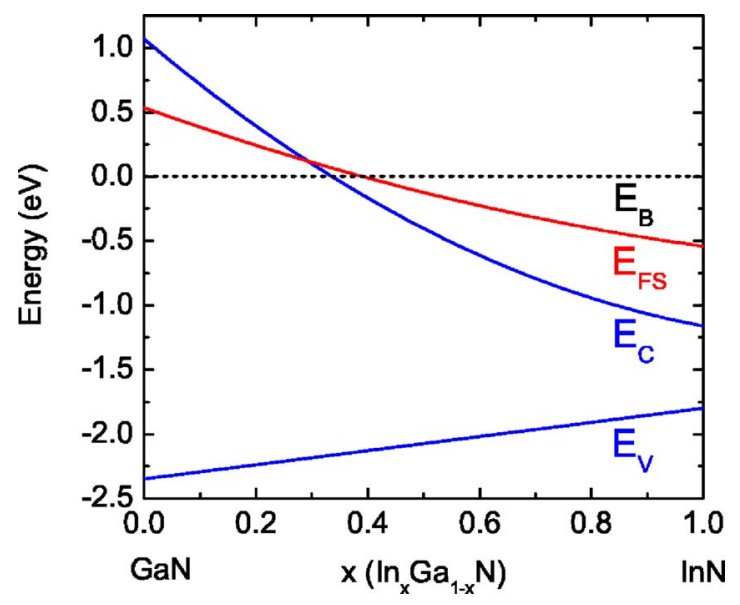

FIG. 3. (Color online) Conduction band edge $\left(E_{C}\right)$ and valence band edge $\left(E_{V}\right)$ of $\operatorname{In}_{x} \mathrm{Ga}_{1-x} \mathrm{~N}$ as a function of $x$ with respect to the universal branchpoint energy $\left(E_{B}\right.$, dashed line). The relative position of the surface Fermi level $\left(E_{\mathrm{FS}}\right)$ as a function of $x$ as determined from the photoemission is also shown. The energy position of $E_{B}$ at $2.35 \mathrm{eV}$ above the VBM in $\mathrm{GaN}$ is taken from the zero-charge-transfer Schottky barrier height (Refs. 5 and 16). This was determined from measurements of different metal-GaN Schottky barrier heights, combined with the concept of charge transfer across the interface between materials of different electronegativities (Refs. 5 and 16). This value is in agreement with the value of $2.37 \mathrm{eV}$ calculated by Mönch (Ref. 17). For InN, $E_{B}$ is located at $\sim 1.8 \mathrm{eV}$ above the VBM, based on previous studies of InN surface electron accumulation (Ref. 2), high energy particle irradiation studies (Ref. 18), and the calculated value of $1.87 \mathrm{eV}$ (Ref. 19). The difference between the $E_{B}$ values in these two end points of the InGaN alloy is therefore $0.55 \mathrm{eV}$ and corresponds to the valence band offset (VBO) between InN and GaN. While there is one report of an $\mathrm{InN} / \mathrm{GaN} \mathrm{VBO}$ as high as $1.05 \mathrm{eV}$ (Ref. 20), other experimental and theoretical studies give a value of $0.5 \mathrm{eV}$ (Refs. 21-23), very close to the difference in $E_{B}$ values. The band gap bowing is shown entirely in the conduction band as $E_{B}$ with respect to the VBM and is known to vary linearly with alloy composition (Ref. 17).

function of depth, ${ }^{25}$ resulting in the XPS tending to slightly underestimate the surface Fermi level. For the GaN, the free electron density is significantly lower and the screening length is correspondingly longer, making the XPS-measured surface Fermi level a good estimate.

In conclusion, the composition dependence of the Fermi level at the surface of $\operatorname{In}_{x} \mathrm{Ga}_{1-x} \mathrm{~N}$ alloys has been determined by XPS. The transition from positive to negative "barrier height" occurs at $x=0.29$. The transition from surface electron depletion to accumulation is expected to occur at a similar composition but additionally depends on the bulk Fermi level of particular samples. The observed surface Fermi level positions are consistent with the concept of interface induced gap states and the literature values for the branch-point en- ergies in $\mathrm{InN}$ and $\mathrm{GaN}$ and the $\mathrm{InN} / \mathrm{GaN}$ valence band offset (VBO).

The authors are grateful to Danny Law and Graham Beamson of NCESS for their assistance with the XPS experiments. The Engineering and Physical Sciences Research Council, UK is acknowledged for access to the NCESS facility under Grant No. GR/S14252/01 and for financial support under Grant No. EP/C535553/01.

${ }^{1}$ Hai Lu, W. J. Schaff, L. F. Eastman, and C. E. Stutz, Appl. Phys. Lett. 82, 1736 (2003).

${ }^{2}$ I. Mahboob, T. D. Veal, C. F. McConville, Hai Lu, and W. J. Schaff, Phys. Rev. Lett. 92, 036804 (2004).

${ }^{3}$ C. A. Mead and W. G. Spitzer, Phys. Rev. 134, A713 (1964).

${ }^{4}$ K. Kajiyama, Y. Mizushima, and S. Sakata, Appl. Phys. Lett. 23, 458 (1973).

${ }^{5}$ H. Luth, Phys. Status Solidi A 187, 33 (2001).

${ }^{6}$ G. Margaritondo, Rep. Prog. Phys. 62, 765 (1999).

${ }^{7}$ V. M. Bermudez, T. M. Jung, K. Doverspike, and A. E. Wickenden, J. Appl. Phys. 79, 101 (1996).

${ }^{8}$ L. F. J. Piper, T. D. Veal, M. Walker, I. Mahboob, C. F. McConville, Hai Lu, and W. J. Schaff, J. Vac. Sci. Technol. A 23, 617 (2005).

${ }^{9}$ Hai Lu, W. J. Schaff, and L. F. Eastman, J. Appl. Phys. 96, 3577 (2004).

${ }^{10}$ W. J. Schaff, Hai Lu, L. F. Eastman, W. Walukiewicz, K. M. Yu, S. Keller, S. Kurtz, B. Keyes, and L. Gevilas, in State-of-the-Art Program on Compound Semiconductors XLI and Nitride and Wide Bandgap Semiconductors for Sensors, Photonics, and Electronics V, The Electrochemical Society Proceedings Series, Honolulu, Hawaii, 3-8 October 2004, edited by H. M. Ng and A. G. Baca (Electrochemical Society, Pennington, NJ, 2004), Vol. 2004-06, pp. 358-371.

${ }^{11}$ S. A. Chambers, T. Droubay, T. C. Kaspar, and M. Gutowski, J. Vac. Sci. Technol. B 22, 2205 (2004).

${ }^{12}$ K. A. Rickert, A. B. Ellis, F. J. Himpsel, Hai Lu, W. Schaff, J. M. Redwing, F. Dwikusuma, and T. F. Kuech, Appl. Phys. Lett. 82, 3254 (2003).

${ }^{13}$ V. Cimalla, M. Niebelschütz, G. Ecke, V. Lebedev, O. Ambacher, M. Himmerlich, S. Krischok, J. A. Schaefer, Hai Lu, and W. J. Schaff, Phys. Status Solidi A 203, 59 (2006).

${ }^{14}$ J. Wu, W. Walukiewicz, K. M. Yu, J. W. Ager III, E. E. Haller, Hai Lu, and W. J. Schaff, Appl. Phys. Lett. 80, 4741 (2002).

${ }^{15}$ S.-J. Cho, S. Dogan, S. Sabuktagin, M. A. Reshchikov, D. K. Johnstone, and H. Morkoc, Appl. Phys. Lett. 84, 3070 (2004).

${ }^{16}$ T. U. Kampen and W. Mönch, Appl. Surf. Sci. 117/118, 388 (1997).

${ }^{17}$ W. Mönch, J. Appl. Phys. 80, 5076 (1996).

${ }^{18}$ S. X. Li, K. M. Yu, J. Wu, R. E. Jones, W. Walukiewicz, J. W. Ager III, W. Shan, E. E. Haller, Hai Lu, and W. J. Schaff, Physica B 376-377, 432 (2006).

${ }^{19}$ J. Robertson and B. Falabretti, J. Appl. Phys. 100, 014111 (2006).

${ }^{20}$ G. Martin, A. Botchkarev, A. Rockett, and H. Morkoc, Appl. Phys. Lett. 68, 2541 (1996).

${ }^{21}$ I. Vurgaftman and J. R. Meyer, J. Appl. Phys. 94, 3675 (2003).

${ }^{22}$ C-F. Shih, N-C. Chen, P-H. Chang, and K-S. Liu, Jpn. J. Appl. Phys., Part 1 44, 7892 (2005).

${ }^{23}$ S.-H. Wei and A. Zunger, Appl. Phys. Lett. 69, 2719 (1996).

${ }^{24} \mathrm{~W}$. Mönch, Electronic Properties of Semiconductor Interfaces (Springer, Berlin, 2004), pp. 107-134.

${ }^{25}$ L. F. J. Piper, T. D. Veal, I. Mahboob, C. F. McConville, Hai Lu, and W. J. Schaff, Phys. Rev. B 70, 115333 (2004). 